QUARTERLY OF APPLIED MATHEMATICS

VOLUME LXIII, NUMBER 2

JUNE 2005, PAGES 343-367

S $0033-569 X(05) 00964-6$

Article electronically published on April 19, 2005

\title{
THE INCOMPLETENESS OF THE BORN-INFELD MODEL FOR NON-LINEAR MULTI-D MAXWELL'S EQUATIONS
}

\author{
$\mathrm{BY}$ \\ WLADIMIR NEVES (Instituto de Matematica, Universidade Federal do Rio de Janeiro, C. Postal \\ 68530, Rio de Janeiro, RJ 21945-970, Brazil) \\ AND \\ DENIS SERRE (UMPA, Ecole Normale Superieure de Lyon, UMR 5669 CNRS, Lyon Cedex 07, \\ France)
}

\begin{abstract}
We study the Born-Infeld system of conservation laws, which is the most famous model for non-linear Maxwell's equations. This system is totally linear degenerated and there exists a conjecture, see Y. Brenier, Hydrodynamic structure of the augmented Born-Infeld equations, Arch. Rational Mech. Anal. 172 (2004), 65-91, that shocks are not allowed to form. In fact, we show that this conjecture is false and that the Born-Infeld model is not complete by itself. It means that a further theory is needed to complete the model.
\end{abstract}

1. Introduction. We are concerned in this paper with the Born-Infeld system of conservation laws, which has come into great discussion recently with the papers of Y. Brenier [4] and D. Serre [20]. This system is certainly the most famous model for nonlinear Maxwell's equations and, since it is totally linear degenerated (see Definition 1.2), there exists a conjecture that shock waves would not form. We show that this conjecture is false, by the appearance of shocks beyond the contact discontinuities in the resolution of the Riemann Problem, at least when the initial-data are large. Moreover, when a rarefaction shock (see Definition 1.3) is present, we show that the Riemann Problem is not well-posed. In fact, we have a one parameter family of solutions for this problem and, in this way, the Born-Infeld model is not complete by itself. Therefore, for this class of Riemann Problems, we need a further local theory to complete the model.

It is well known that, when a system of conservation laws is endowed with a renormalized equation given by a uniformly convex entropy, the initial-value problem is locally well-posed in the context of classical solutions; see Majda [18, Dafermos [10]. Furthermore, we have uniqueness and continuous dependence on the initial-data for a broader

Received October 5, 2004.

2000 Mathematics Subject Classification. Primary 35Q60.

E-mail address: wladimir@im.ufrj.br

E-mail address: serre@umpa.ens-lyon.fr

(C)2005 Brown University 
class of weak solutions that satisfies an admissibility inequality constraint; see [11, 12] and also Bressan et al. [5, 6, 7, 8] in the BV case. For instance, this is the case of piecewise smooth solutions in the class of Riemann Problems. On the other hand, the classical problem of elastodynamics in nonlinear elasticity shows that the uniqueness of solution for the Riemann Problem is lost when there exists a lack of convexity on the entropy function; see for example [1, 10, 15, 17] and many other references therein. This difficulty was resolved in 1, 17, by considering principally a further local theory given by a kinetic formulation. However, [10] overcame this problem of non-uniqueness for elastodynamics by considering extra conservation laws, called involutions, which compensate for the lack of convexity. Analogously, it is the problem in fluid-dynamics for van der Waals fluid; see [15].

Here we have the same condition, that is, lack of convexity for the energy function, which is the entropy for the Born-Infeld model. As we shall see, the energy is strictly convex in a neighborhood of the origin, but is not far away from it. Hence, the BornInfeld system is not hyperbolic in the large. Due to this lack of convexity, we are not allowed to discard some nonphysical types of jump discontinuities as non-classical shocks. Actually, they are a potential source of non-uniqueness, which is the case above and for the Born-Infeld system of equations.

Finally, we mention the idea of an enlarged system of Born-Infeld equations introduced in 4, 20, following [10, 13] in the case of nonlinear elasticity. In [20], the polyconvexity of the energy (entropy) function was a sufficient condition to prove local well-posedness of the initial-value problem for classical solutions, that is, for initial-data in $H^{s}$, with $s>1+d / 2$, where $d$ is the dimension of the space. However, the Rankine-Hugoniot condition for the enlarged system is not equivalent to the Born-Infeld one. Therefore, a couple of states, which satisfies the Rankine-Hugoniot condition for the Born-Infeld model, does not necessarily satisfy the enlarged one. Moreover, the enlarged system could suggest a local kinetic formulation in the class of Riemann Problems. We address these interesting questions in other works.

Let $(t, x) \in \mathbb{R}^{+} \times \mathbb{R}^{3}$ be the points in the time-space domain. We consider the electric intensity field $E$, the magnetic intensity field $H$, the electric induction $D$, and the magnetic induction $B$, all of them taking values in $\mathbb{R}^{3}$. The models of electromagnetism could be given in a rational continuum physics form through the stored energy function $h(D, B)$, see Coleman and Dill [9], by the following form:

$$
\begin{aligned}
& \partial_{t} B+\operatorname{curl}_{x}\left(\partial_{D} h\right)=0, \\
& \partial_{t} D-\operatorname{curl}_{x}\left(\partial_{B} h\right)=0, \\
& \operatorname{div}_{x} D=0, \quad \operatorname{div}_{x} B=0,
\end{aligned}
$$

where we have assumed for simplicity that there are neither charges nor currents. Furthermore, we have

$$
\partial_{D_{i}} h(D, B)=: E_{i}, \quad \partial_{B_{i}} h(D, B)=: H_{i} \quad(i=1, \ldots, 3) .
$$


The equations (1.1), (1.2) are respectively the Faraday and Ampere's Law, and equation (1.3) are constraints, which are compatible with (1.1), (1.2). In the linear theory of electromagnetism, called Maxwell's equations, we have

$$
D=\epsilon E \quad \text { and } \quad B=\mu H
$$

where $\epsilon$ is the dielectric tensor and $\mu$ is the permeability tensor. However, there are several reasons to avoid the linear case. For instance, the electric field of a particle at rest decreases with the inverse square of the distance, which means that $E$ grows without limit as the distance tends to zero. To get rid of this fact, some non-linear models have been proposed; the most famous is due to M. Born and L. Infeld [3]. This model is obtained by taking

$$
h(D, B)=\sqrt{1+|D|^{2}+|B|^{2}+|P|^{2}} \quad(P:=D \times B) .
$$

From (1.1), (1.2) and (1.4), we obtain

$$
\begin{gathered}
\partial_{t} D+\operatorname{curl}_{x}\left(\frac{-B+D \times P}{h}\right)=0, \\
\partial_{t} B+\operatorname{curl}_{x}\left(\frac{D+B \times P}{h}\right)=0 .
\end{gathered}
$$

Then, (1.3), (1.5) and (1.6) consist of the Born-Infeld system of conservation laws in 3dimensions. Moreover, the stored energy function $h$ given by (1.4) satisfies an additional conservation law, that is

$$
\partial_{t} h+\operatorname{div}_{x} P=0 .
$$

Then, $(h, P)$ is an entropy pair (see Definition 1.1) for the Born-Infeld system. We note that $h(D, B)$ is a strictly convex function of $D$ and $B$ only in a neighborhood of the origin, but fails to be convex far away from it. The entropy flux $P$ is usually called the Poynting vector.

Now we focus on plane waves, which depend only on the time and one scalar space variable. We observe that the stored energy function which gives the Born-Infeld model describes an isotropic medium. In fact, this behavior happens since $h$ derives from a Lorentz- and orientation-invariant Lagrangian, i.e.,

$$
L=-\sqrt{1+|B|^{2}-|E|^{2}-(E \cdot B)^{2}} .
$$

Therefore, we have a wave isotropy condition, see [16], and by symmetry considerations thus far, only a single spatial variable is needed. Let us choose $x=x_{1}$ as such a spatial coordinate. Hence, all the fields involved in (1.3)-(1.6) depend on $(t, x) \in \mathbb{R}^{+} \times \mathbb{R}$. Moreover, it follows that

$$
\begin{aligned}
& \partial_{t} D_{1}=0, \quad \partial_{x} D_{1}=0, \\
& \partial_{t} B_{1}=0, \quad \partial_{x} B_{1}=0 .
\end{aligned}
$$


Thus, $D_{1}, B_{1}$ are constant functions, and for simplicity, we assume $D_{1}=B_{1}=0$. Then we obtain the following system of conservation laws:

$$
\begin{gathered}
\partial_{t} D_{2}+\partial_{x}\left(\frac{B_{3}+D_{2} P}{h}\right)=0, \\
\partial_{t} D_{3}+\partial_{x}\left(\frac{-B_{2}+D_{3} P}{h}\right)=0, \\
\partial_{t} B_{2}+\partial_{x}\left(\frac{-D_{3}+B_{2} P}{h}\right)=0, \\
\partial_{t} B_{3}+\partial_{x}\left(\frac{D_{2}+B_{3} P}{h}\right)=0,
\end{gathered}
$$

where $P \equiv P_{1}=D_{2} B_{3}-D_{3} B_{2}$.

Next we present some mathematical considerations for systems of conservation laws. Set

$$
\begin{gathered}
u:=\left(D_{2}, D_{3}, B_{2}, B_{3}\right), \\
f(u):=\left(\frac{B_{3}+D_{2} P}{h}, \frac{-B_{2}+D_{3} P}{h}, \frac{-D_{3}+B_{2} P}{h}, \frac{D_{2}+B_{3} P}{h}\right) .
\end{gathered}
$$

So (1.8)-1.11) could be written in the following simple form:

$$
u_{t}+f(u)_{x}=0 \quad \text { in } \mathbb{R}^{+} \times \mathbb{R} .
$$

The open set $U \subset \mathbb{R}^{4}$, such that $u(t, x) \in U$ will be called the set of states and $f$ the flux function. We are concerned with the initial-value problem, that is, we seek a $u(t, x) \in U$ solution of (1.14) and satisfying an initial-data

$$
u(0, x)=u_{0} \quad x \in \mathbb{R},
$$

where $u_{0}: \mathbb{R} \rightarrow U$ is a given bounded measurable function. As is well known, in general for conservation laws, there does not exist (global) solutions, even if the data is infinitely differentiable. Hence, we have to deal with the concept of weak solutions, which means that $u \in L^{\infty}\left(\mathbb{R}^{+} \times \mathbb{R} ; \mathbb{R}^{4}\right)$ is a weak solution of (1.14), (1.15) if it satisfies

$$
\int_{0}^{\infty} \int_{\mathbb{R}}(u, f(u)) \cdot \nabla_{t, x} \phi(t, x) d x d t+\int_{\mathbb{R}} u_{0} \phi(0, x) d x=0,
$$

for any function $\phi \in C_{0}^{\infty}\left(\mathbb{R}^{2}\right)$. Moreover, if $u \in L^{\infty}$ is a $C^{1}$ function outside a manifold $\Gamma$ (with codimension one), across which it has jump discontinuities, then it can be shown using (1.16), see [10, 19], that $u$ must satisfy the so-called Rankine-Hugoniot condition

$$
n_{t}[u]+n_{x}[f(u)]=0,
$$

where $n=\left(n_{t}, n_{x}\right)$ is the outward unit normal vector along the manifold $\Gamma,[u]:=u^{+}-u^{-}$, $[f(u)]:=f\left(u^{+}\right)-f\left(u^{-}\right)$, and

$$
u^{+}=\lim _{\delta \rightarrow 0^{+}} u((t, x)+\delta n), \quad u^{-}=\lim _{\delta \rightarrow 0^{+}} u((t, x)-\delta n) .
$$


Definition 1.1. A real Lipschitz function $\eta$ is called an entropy for (1.14), with associated entropy flux $q \in W^{1, \infty}(U)$, when for every open set $\Pi \subset \mathbb{R}^{+} \times \mathbb{R}$ and for every $u \in C^{1}$ that solves (1.14) pointwise, we have

$$
\partial_{t} \eta(u)+\partial_{x} q(u)=0 \quad \text { in } \mathcal{D}^{\prime}(\Pi) .
$$

If, in addition, $\eta$ is a convex function, then we say that $(\eta, q)$ is a convex entropy pair. Moreover, a weak solution of (1.14), (1.15) is called an entropy solution when $\partial_{t} \eta(u)+$ $\partial_{x} q(u) \leq 0$ in the sense of distributions for every convex entropy pair.

We recall that (see [10 19]) a system of conservation laws is said to be hyperbolic if for any $v \in U$, the matrix

$$
A_{i, j}(v):=\frac{\partial f_{i}(v)}{\partial v_{j}} \quad(i, j=1, \ldots, n),
$$

has $n$ real eigenvalues $\lambda_{1}(v) \leq \lambda_{2}(v) \leq \ldots \leq \lambda_{n}(v)$ and is diagonalizable. Thus, there exist $r_{i}(v),(i=1, \ldots, n)$ linearly independent (right) corresponding eigenvectors, and

$$
A(v) r_{i}(v)=\lambda_{i}(v) r_{i}(v) .
$$

Here, $n=4$ and this computation is hard to derive. Furthermore, since $h$ is not convex in the large, it does not follow from the well-known result that the existence of a convex entropy pair for (1.14) implies the hyperbolicity. Although, taking account of the augmented theory (see [4), the propagation speeds, i.e., $\lambda$ 's, are easily calculated. We have

$$
\lambda_{1}=\lambda_{2}=\frac{P-1}{h}=: \lambda^{-}<\lambda_{3}=\lambda_{4}=\frac{P+1}{h}=: \lambda^{+} .
$$

Definition 1.2. For the system of conservation laws (1.14), a point $v \in U$ is said to be of linear degeneracy of the $i$-characteristic family when

$$
\nabla_{v} \lambda_{i}(v) \cdot r_{i}(v)=0
$$

otherwise, it is of genuine nonlinearity of the $i$-characteristic family. If (1.18) holds for every $v \in U$, then the $i$-characteristic family is called linear degenerated. Moreover, we say that (1.14) is totally linear degenerated when every $i$-characteristic is linear degenerated.

Again, it is not easy to conclude from the above definition that the Born-Infeld model is totally linear degenerated. However, from (1.17), it is an immediate application of Boillat's theorem; see [2].

In fact, for the study of shocks and completeness of the Born-Infeld model, we take the Riemann Problem. Thus, we consider initial-data of the form

$$
u_{0}(x)= \begin{cases}u_{0}^{\ell} & \text { if } x<0 \\ u_{0}^{r} & \text { if } x>0\end{cases}
$$

where $u_{0}^{\ell}, u_{0}^{r}$ are given constants. We seek self-similar solutions for

$$
v(\xi)=u(t, x) \quad\left(\xi=\frac{x}{t}\right)
$$


in $B V_{\text {loc }}\left(\mathbb{R} ; \mathbb{R}^{4}\right) \cap L^{\infty}\left(\mathbb{R} ; \mathbb{R}^{4}\right)$, satisfying in the sense of distributions the ordinary differential equation

$$
[\xi v(\xi)-f(v(\xi))]^{\prime}=v(\xi) \quad\left({ }^{\prime} \equiv \frac{d}{d \xi}\right)
$$

and the boundary conditions

$$
v(-\infty)=u_{0}^{\ell}, \quad v(+\infty)=u_{0}^{r} .
$$

In fact, $v$ is a Lipschitz function, and thus by Rademacher's Theorem it is differentiable $\mathcal{L}^{1}$-a.e.; see [14]. Hence, (1.20) is satisfied by:

i) Constant states; for each Lebesgue point $\xi$, where

$$
v^{\prime}(\xi)=0 .
$$

ii) Jump discontinuities; for each discontinuity point $\xi$, where the Rankine-Hugoniot jump condition must hold, i.e.,

$$
\xi\left[v^{+}-v^{-}\right]=f\left(v^{+}\right)-f\left(v^{-}\right) \quad\left(v^{+}:=v\left(\xi^{+}\right), v^{-}:=v\left(\xi^{-}\right)\right) .
$$

iii) Centered simple waves; for each Lebesgue point $\xi$, where $v^{\prime}(\xi) \neq 0$. From (1.20), i.e., $\left[D f(v(\xi))-\xi I_{d}\right] v^{\prime}(\xi)=0$, we must have

$$
\xi=\lambda_{i}(v(\xi)) \text { and } \quad v^{\prime}(\xi)=c(\xi) r_{i}(v(\xi)) \quad(i=1, \ldots, 4) .
$$

Moreover, if we set

$$
\begin{aligned}
& \mathcal{C}:=\left\{\xi \in \mathbb{R} ; v^{\prime}(\xi)=0\right\}, \\
& \mathcal{J}:=\{\xi \in \mathbb{R} ; \text { the Rankine-Hugoniot condition holds }\}, \\
& \mathcal{W}:=\left\{\xi \in \mathbb{R} ; v^{\prime}(\xi) \neq 0\right\},
\end{aligned}
$$

then $\mathbb{R}$ is the union of these pairwise disjoint sets. Therefore, the solutions $v(\xi)$ of $(1.20)$, (1.21) are given by a combination of $(i)-(i i i)$.

REMARK 1.1. By differentiating the first relation in (1.22) and utilizing the second, we obtain

$$
\left[D \lambda_{i}(v(\xi)) \cdot r_{i}(v(\xi))\right] c(v(\xi))=1 .
$$

Since $v^{\prime}$ is a locally finite Radon measure, we observe that the centered simple waves are points of genuine nonlinearity of the $i$-characteristic family. Moreover, from the above expression, we determine the scalar function $c$. Therefore, for totally linear degenerated systems of conservation laws, we have $\mathcal{W}=\emptyset$.

Usually, the jump $v^{+}-v^{-}$is called the amplitude and its size $\left|v^{+}-v^{-}\right|$is the strength of the jump discontinuity. Moreover, when the strength of the jump discontinuity is less than a positive (sufficiently small) $\delta$, we say that the jump discontinuity is weak.

DeFinition 1.3. We say that the jump discontinuity $\left(v^{-}, v^{+} ; \xi\right)$ is an $i$-classical shock (or $i$-Lax shock, or $i$-compressive shock), when there exists an index $i(1 \leq i \leq n)$ such that

$$
\begin{gathered}
\lambda_{i}\left(v^{+}\right)<\xi<\lambda_{i}\left(v^{-}\right), \\
\lambda_{i-1}\left(v^{-}\right)<\xi<\lambda_{i+1}\left(v^{+}\right) .
\end{gathered}
$$


It means that at the point of discontinuity, there are $n+1$ incoming characteristics, of which the speeds are the eigenvalues

$$
\lambda_{1}\left(v^{+}\right), \ldots, \lambda_{i}\left(v^{+}\right), \lambda_{i}\left(v^{-}\right), \ldots, \lambda_{n}\left(v^{-}\right) .
$$

Moreover, (1.23) is called the Lax shock admissibility criterion. When the left or the right part of $(1.23)_{1}$ is satisfied as equality, the jump discontinuity is called a left or a right $i$-contact discontinuity, and if both parts hold as equalities, then we have an $i$-contact discontinuity. When there are at least $n+2$ incoming characteristics, the jump discontinuity $\left(v^{-}, v^{+} ; \xi\right)$ is called an $i$-overcompressive shock; that is, there exists an index $i$ such that

$$
\lambda_{i+1}\left(v^{+}\right)<\xi<\lambda_{i}\left(v^{-}\right) .
$$

When there are $n$ incoming characteristics, the jump discontinuity $\left(v^{-}, v^{+} ; \xi\right)$ is called an $i$-undercompressive shock (or $i$-transitional shock); that is, there exists an index $i$ such that

$$
\lambda_{i}\left(v^{ \pm}\right)<\xi<\lambda_{i+1}\left(v^{ \pm}\right) .
$$

When there are $n-1$ incoming characteristics, the jump discontinuity $\left(v^{-}, v^{+} ; \xi\right)$ is called an $i$-rarefaction shock (or $i$-counter Lax shock); that is, there exists an index $i$ such that

$$
\begin{gathered}
\lambda_{i}\left(v^{-}\right)<\xi<\lambda_{i}\left(v^{+}\right), \\
\lambda_{i-1}\left(v^{+}\right)<\xi<\lambda_{i+1}\left(v^{-}\right) .
\end{gathered}
$$

In any (1.24)-1.26) case, we say that the jump discontinuity is a non-classical shock.

2. Propagation speeds for the Born-Infeld model. The aim of this section is to study the Born-Infeld model, when given two constant states

$$
u^{\ell}=\left(D_{2}^{\ell}, D_{3}^{\ell}, B_{2}^{\ell}, B_{3}^{\ell}\right), \quad u^{r}=\left(D_{2}^{r}, D_{3}^{r}, B_{2}^{r}, B_{3}^{r}\right),
$$

not necessarily close, or small, how they could be connected. Since the Born-Infeld system of equations is totally linear degenerated, from Remark 1.1 we are not allowed to use centered simple waves. So, we have to connect $u^{\ell} \equiv u^{-}$and $u^{r} \equiv u^{+}$by jump discontinuities. Therefore, for any $s:=\xi \in \mathcal{J}$, we regard the Rankine-Hugoniot jump condition given from (1.8) 1.11 ; that is

$$
\begin{gathered}
s\left(D_{2}^{+}-D_{2}^{-}\right)=\frac{B_{3}^{+}+D_{2}^{+} P^{+}}{h^{+}}-\frac{B_{3}^{-}+D_{2}^{-} P^{-}}{h^{-}}, \\
s\left(D_{3}^{+}-D_{3}^{-}\right)=\frac{-B_{2}^{+}+D_{3}^{+} P^{+}}{h^{+}}-\frac{-B_{2}^{-}+D_{3}^{-} P^{-}}{h^{-}}, \\
s\left(B_{2}^{+}-B_{2}^{-}\right)=\frac{-D_{3}^{+}+B_{2}^{+} P^{+}}{h^{+}}-\frac{-D_{3}^{-}+B_{2}^{-} P^{-}}{h^{-}}, \\
s\left(B_{3}^{+}-B_{3}^{-}\right)=\frac{D_{2}^{+}+B_{3}^{+} P^{+}}{h^{+}}-\frac{D_{2}^{-}+B_{3}^{-} P^{-}}{h^{-}},
\end{gathered}
$$

where $h, P$ are given by (1.4) with the obvious notations for \pm . Now, if we set

$$
d_{i}^{ \pm}:=\frac{D_{i}^{ \pm}}{h^{ \pm}}, \quad b_{i}^{ \pm}:=\frac{B_{i}^{ \pm}}{h^{ \pm}} \quad(i=2,3),
$$


then we can rewrite the above equations as

$$
\begin{aligned}
& d_{2}^{+} \zeta^{+}-d_{2}^{-} \zeta^{-}-b_{3}^{+}+b_{3}^{-}=0, \\
& d_{3}^{+} \zeta^{+}-d_{3}^{-} \zeta^{-}+b_{2}^{+}-b_{2}^{-}=0, \\
& b_{2}^{+} \zeta^{+}-b_{2}^{-} \zeta^{-}+d_{3}^{+}-d_{3}^{-}=0, \\
& b_{3}^{+} \zeta^{+}-b_{3}^{-} \zeta^{-}-d_{2}^{+}+d_{2}^{-}=0,
\end{aligned}
$$

where $\zeta^{ \pm}=s h^{ \pm}-P^{ \pm}$. So instead of $s$, we have two unknowns, i.e., $\zeta^{ \pm}$. Hence, we obtain one more equation to be satisfied:

$$
\phi\left(\zeta^{+}, \zeta^{-}\right):=\frac{P^{+}+\zeta^{+}}{h^{+}}-\frac{P^{-}+\zeta^{-}}{h^{-}}=0,
$$

which means that $s$ must have the same value given by

$$
\zeta^{+}=s h^{+}-P^{+} \quad \text { or } \quad \zeta^{-}=s h^{-}-P^{-} .
$$

Once we obtain $\zeta^{+}, \zeta^{-}$satisfying (2.1)-(2.5), the Rankine-Hugoniot condition is satisfied. Moreover, it follows from $\{(2.1)-(2.4)\}$ and $\{(2.2)+(2.3)\}$ that

$$
\left[\begin{array}{ll}
\left(d_{2}^{+}-b_{3}^{+}\right) & -\left(d_{2}^{-}-b_{3}^{-}\right) \\
\left(d_{3}^{+}+b_{2}^{+}\right) & -\left(d_{3}^{-}+b_{2}^{-}\right)
\end{array}\right]\left[\begin{array}{c}
\left(\zeta^{+}+1\right) \\
\left(\zeta^{-}+1\right)
\end{array}\right]=0 .
$$

Analogously, from $\{(2.1)+2.4\}$ and $\{2.2 .2 .3\}$, we have

$$
\left[\begin{array}{ll}
\left(d_{2}^{+}+b_{3}^{+}\right) & -\left(d_{2}^{-}+b_{3}^{-}\right) \\
\left(d_{3}^{+}-b_{2}^{+}\right) & -\left(d_{3}^{-}-b_{2}^{-}\right)
\end{array}\right]\left[\begin{array}{c}
\left(\zeta^{+}-1\right) \\
\left(\zeta^{-}-1\right)
\end{array}\right]=0 .
$$

Hence, we can equivalently search for $\zeta^{ \pm}$satisfying (2.5)-(2.7). We begin regarding the values

$$
\begin{aligned}
& \zeta^{+}=\zeta^{-}=-1 \\
& \zeta^{+}=\zeta^{-}=+1 .
\end{aligned}
$$

The former trivially solves (2.6), and from (2.7) we must have

$$
\frac{D_{2}^{+}+B_{3}^{+}}{h^{+}}=\frac{D_{2}^{-}+B_{3}^{-}}{h^{-}} \text {and } \frac{D_{3}^{+}-B_{2}^{+}}{h^{+}}=\frac{D_{3}^{-}-B_{2}^{-}}{h^{-}} .
$$

From (2.8), we obtain

$$
\frac{\left(D_{2}^{+}\right)^{2}+2 D_{2}^{+} B_{3}^{+}+\left(B_{3}^{+}\right)^{2}}{\left(h^{+}\right)^{2}}=\frac{\left(D_{2}^{-}\right)^{2}+2 D_{2}^{-} B_{3}^{-}+\left(B_{3}^{-}\right)^{2}}{\left(h^{-}\right)^{2}}
$$

and

$$
\frac{\left(D_{3}^{+}\right)^{2}-2 D_{3}^{+} B_{2}^{+}+\left(B_{2}^{+}\right)^{2}}{\left(h^{+}\right)^{2}}=\frac{\left(D_{3}^{-}\right)^{2}-2 D_{3}^{-} B_{2}^{-}+\left(B_{2}^{-}\right)^{2}}{\left(h^{-}\right)^{2}} .
$$

Adding the two above equations, we have

$$
\frac{\left|D^{+}\right|^{2}+\left|B^{+}\right|^{2}+2 P^{+}}{\left(h^{+}\right)^{2}}=\frac{\left|D^{-}\right|^{2}+\left|B^{-}\right|^{2}+2 P^{-}}{\left(h^{-}\right)^{2}},
$$


where we have used the definition of $P$ given by (1.4). Hence, after some algebra and the definition of $h$ given by (1.4), we obtain

$$
1-\frac{\left(P^{+}-1\right)^{2}}{\left(h^{+}\right)^{2}}=1-\frac{\left(P^{-}-1\right)^{2}}{\left(h^{-}\right)^{2}} .
$$

Therefore, it follows that

$$
\frac{P^{+}-1}{h^{+}}-\frac{P^{-}-1}{h^{-}}=0
$$

or

$$
\frac{P^{+}-1}{h^{+}}+\frac{P^{-}-1}{h^{-}}=0 .
$$

In order to satisfy (2.5), we must have

$$
\frac{P^{-}-1}{h^{-}}=\frac{P^{+}-1}{h^{+}} \quad(=s) .
$$

For the second case, that is $\zeta^{+}=\zeta^{-}=+1$, we have (2.7) trivially satisfied, and from $(2.6)$, we must have

$$
\frac{D_{2}^{+}-B_{3}^{+}}{h^{+}}=\frac{D_{2}^{-}-B_{3}^{-}}{h^{-}} \text {and } \frac{D_{3}^{+}+B_{2}^{+}}{h^{+}}=\frac{D_{3}^{-}+B_{2}^{-}}{h^{-}} .
$$

Analogously, (2.9) and (1.4) imply

$$
\frac{P^{-}+1}{h^{-}}=\frac{P^{+}+1}{h^{+}} \quad(=s) .
$$

Therefore, considering the BI system of conservation laws given by (1.8)-(1.11), we have the following:

Lemma 2.1. Let $u^{-}=\left(D_{2}^{-}, D_{3}^{-}, B_{2}^{-}, B_{3}^{-}\right), u^{+}=\left(D_{2}^{+}, D_{3}^{+}, B_{2}^{+}, B_{3}^{+}\right)$be two given constant states. Let (1.8), (1.11) be the system of conservation laws for $u=(D, B)$. Then $u^{-}, u^{+}$could be connected by contact discontinuities in the following form:

i) When $\left(u^{-}, u^{+}\right)$satisfies $(2.8)$, by a contact discontinuity of speed

$$
s=\lambda^{-}\left(u^{-}\right)=\lambda^{-}\left(u^{+}\right) .
$$

ii) When $\left(u^{-}, u^{+}\right)$satisfies (2.9), by a contact discontinuity of speed

$$
s=\lambda^{+}\left(u^{-}\right)=\lambda^{+}\left(u^{+}\right) .
$$

REMARK 2.1. We recall the well-known result that any weak jump discontinuity associated with a linear degenerated characteristic family is necessarily a contact discontinuity (see [10, 19]). Moreover, any two nearby states $u^{-}$and $u^{+}$associated with a linear degenerated $i$-characteristic family could be connected to each other by a contact discontinuity of speed

$$
s=\lambda_{i}\left(u^{-}\right)=\lambda_{i}\left(u^{+}\right) .
$$

Now, we study whether there exists $\zeta^{ \pm} \neq \pm 1$ satisfying (2.5)-(2.7). We must have

$$
\begin{aligned}
& \left(d_{3}^{+}+b_{2}^{+}\right)\left(d_{2}^{-}-b_{3}^{-}\right)-\left(d_{2}^{+}-b_{3}^{+}\right)\left(d_{3}^{-}+b_{2}^{-}\right)=0, \\
& \left(d_{3}^{+}-b_{2}^{+}\right)\left(d_{2}^{-}+b_{3}^{-}\right)-\left(d_{2}^{+}+b_{3}^{+}\right)\left(d_{3}^{-}-b_{2}^{-}\right)=0,
\end{aligned}
$$


which define a conic manifold $\mathcal{M}$ of dimension 6. Let $\sigma \neq 0, \theta \neq 0, \gamma^{ \pm}, \alpha^{ \pm}$, be real numbers such that

$$
\begin{array}{ll}
\zeta^{+}+1=\gamma^{+} \sigma, & \zeta^{-}+1=\gamma^{-} \sigma, \\
\zeta^{+}-1=\alpha^{+} \theta, & \zeta^{-}-1=\alpha^{-} \theta .
\end{array}
$$

Once (2.10), (2.11) are satisfied, we may obtain $\gamma^{ \pm}, \alpha^{ \pm} \neq 0$ from (2.6) and (2.7) respectively. Moreover, by eliminating $\zeta^{+}$and $\zeta^{-}$in (2.12), we have

$$
\left[\begin{array}{ll}
\gamma^{+} & -\alpha^{+} \\
\gamma^{-} & -\alpha^{-}
\end{array}\right]\left[\begin{array}{l}
\sigma \\
\theta
\end{array}\right]=\left[\begin{array}{l}
2 \\
2
\end{array}\right] .
$$

Consequently, for $\alpha^{+} \gamma^{-}-\alpha^{-} \gamma^{+} \neq 0$, it follows that

$$
\begin{aligned}
& \left(\alpha^{+} \gamma^{-}-\alpha^{-} \gamma^{+}\right) \sigma=2\left(\alpha^{+}-\alpha^{-}\right), \\
& \left(\alpha^{+} \gamma^{-}-\alpha^{-} \gamma^{+}\right) \theta=2\left(\gamma^{+}-\gamma^{-}\right) .
\end{aligned}
$$

So $\gamma^{+} \neq \gamma^{-}$and $\alpha^{+} \neq \alpha^{-}$. Finally, we obtain from (2.12)

$$
\begin{gathered}
\zeta^{+}=\frac{2 \alpha^{+} \gamma^{+}-\alpha^{+} \gamma^{-}-\alpha^{-} \gamma^{+}}{\alpha^{+} \gamma^{-}-\alpha^{-} \gamma^{+}}, \\
\zeta^{-}=\frac{\alpha^{+} \gamma^{-}+\alpha^{-} \gamma^{+}-2 \alpha^{-} \gamma^{-}}{\alpha^{+} \gamma^{-}-\alpha^{-} \gamma^{+}} .
\end{gathered}
$$

Since we seek $\zeta^{ \pm} \neq \pm 1$, we are not allowed to assume that none of $\gamma^{ \pm}, \alpha^{ \pm}$is zero. Although, we are also interested when

$$
\begin{aligned}
& \zeta^{+}= \pm 1 \quad \text { and } \quad \zeta^{-} \neq \pm 1, \\
& \zeta^{+} \neq \pm 1 \quad \text { and } \quad \zeta^{-}= \pm 1,
\end{aligned}
$$

because this could be a solution for (2.5)-(2.7). It could be, when exactly one of the $\gamma^{ \pm}, \alpha^{ \pm}$is zero. Indeed, if for instance $\gamma^{+}=0$, then we must have $\gamma^{-} \neq 0$, and $\alpha^{+} \neq 0$, since

$$
0 \neq \alpha^{+} \gamma^{-}-\alpha^{-} \gamma^{+} \equiv \alpha^{+} \gamma^{-} .
$$

It follows from (2.13), (2.14) respectively that $\zeta^{+}=-1, \zeta^{-}=1-2\left(\alpha^{-} / \alpha^{+}\right)$, and since $\alpha^{-} \neq \alpha^{+}, \zeta^{-} \neq-1$. Now, if we suppose $\alpha^{-}=0$, then it is impossible to satisfy (2.5). Indeed, for $\gamma^{+}=\alpha^{-}=0$, we must have

$$
\begin{aligned}
& \left(d_{2}^{-}-b_{3}^{-}\right)=0, \quad\left(d_{3}^{-}+b_{2}^{-}\right)=0, \\
& \left(d_{2}^{+}+b_{3}^{+}\right)=0, \quad\left(d_{3}^{+}-b_{2}^{+}\right)=0,
\end{aligned}
$$

which imply

$$
\frac{P^{+}-1}{h^{+}}=-\frac{\left|D^{+}\right|^{2}+\left|B^{+}\right|^{2}}{h^{+}}-\frac{1}{h^{+}}<0,
$$

and

$$
\frac{P^{-}+1}{h^{-}}=\frac{\left|D^{-}\right|^{2}+\left|B^{-}\right|^{2}}{h^{-}}+\frac{1}{h^{-}}>0 .
$$


Actually, the problem now is quite different; that is, we want to construct initial-data $u^{-}, u^{+}$to be connected by a jump discontinuity that is not a contact discontinuity. As (2.10), (2.11) are 2 equations for 8 variables, we have a lot of solutions. Let

$$
\left(\delta_{2}, \delta_{3}, \beta_{2}, \beta_{3}\right)^{ \pm}:=\left(\delta_{2}^{-}, \delta_{3}^{-}, \beta_{2}^{-}, \beta_{3}^{-}, \delta_{2}^{+}, \delta_{3}^{+}, \beta_{2}^{+}, \beta_{3}^{+}\right) \in \mathbb{R}^{8}
$$

be one of these solutions. We seek

$$
\left(d_{2}, d_{3}, b_{2}, b_{3}\right)^{ \pm}=\mu\left(\delta_{2}, \delta_{3}, \beta_{2}, \beta_{3}\right)^{ \pm}
$$

with $\mu \in \mathbb{R}^{+}$to be determined a posteriori. Moreover, for any $\mu,\left(d_{2}, d_{3}, b_{2}, b_{3}\right)^{ \pm} \in \mathcal{M}$. We observe that $(d, b)^{ \pm}$must satisfy some conditions in order to yield the states $u^{-}$and $u^{+}$. For simplicity, we drop the superscript \pm , i.e., + and - , whenever indifferent. Once we have $(d, b)$ to obtain $(D, B)$ from it, we need the value of $h$, which is not known. In fact, $h=h(D, B)$, given by (1.4). However, if the following implicit equation for $h$, i.e.,

$$
G(h):=h-H(d, b ; h)=0 \quad(H(d, b ; h) \equiv h(h d, h b)),
$$

is resolved, then we have $h=h(D, B)$, which means that $D=h d$ and $B=h b$. So $X:=h^{2}$ has to be a solution of

$$
p^{2} X^{2}+\left(|b|^{2}+|d|^{2}-1\right) X+1=0,
$$

where $p=d_{2} b_{3}-d_{3} b_{2}$. We suppose temporarily that $p \neq 0$. For positive real roots, we must have

$$
\begin{aligned}
& \left(1-|b|^{2}-|d|^{2}\right)^{2} \geq 4 p^{2}, \\
& 1-|b|^{2}-|d|^{2}>0 .
\end{aligned}
$$

Hence, we obtain the following constraint:

$$
|b|^{2}+|d|^{2}+2|p| \leq 1
$$

which is the intersection of two orthogonal cylinders; that is,

$$
\begin{aligned}
& \left(d_{3}+b_{2}\right)^{2}+\left(b_{3}-d_{2}\right)^{2} \leq 1, \\
& \left(b_{2}-d_{3}\right)^{2}+\left(b_{3}+d_{2}\right)^{2} \leq 1 .
\end{aligned}
$$

Therefore, (2.16) and 2.17) define a convex set, and so a connected one. Moreover, (2.10), (2.11), (2.16), (2.17) are perfect compatible. Now, for $|(d, b)| \ll 1$, the roots of (2.15) are

$$
X_{1} \sim 1, \quad X_{2} \sim \frac{1}{p^{2}} \geq \frac{1}{|b|^{2}|d|^{2}} .
$$

On the other hand, the right-hand side of (2.15) is positive for $X=1$. Consequently, the two roots are on the same side of 1 . By connectedness, they remain in the same interval and, moreover for $|(d, b)| \ll 1$, they are in $(1, \infty)$. Finally:

LEMMA 2.2. If $\left(d_{2}^{-}, d_{3}^{-}, b_{2}^{-}, b_{3}^{-}\right),\left(d_{2}^{+}, d_{3}^{+}, b_{2}^{+}, b_{3}^{+}\right)$satisfy (2.16)-(2.17), then the respective roots of (2.15) belong to $(1, \infty)$, that is

$$
X_{1}^{-}, X_{2}^{-} \in(1, \infty), \quad X_{1}^{+}, X_{2}^{+} \in(1, \infty) .
$$


Set $h_{i}:=X_{i}^{1 / 2}(i=1,2), X_{i}$ being the roots of (2.15), such that

$$
1 \leq X_{1} \leq X_{2}<\infty
$$

Therefore, making $D^{i}=h_{i} d, B^{i}=h_{i} b$, we obtain

$$
h_{i}^{2}=1+\left|B^{i}\right|^{2}+\left|D^{i}\right|^{2}+P_{i}^{2} \quad(i=1,2),
$$

where $P_{i}:=D_{2}^{i} B_{3}^{i}-D_{3}^{i} B_{2}^{i}$. So, $h_{i}=h\left(D^{i}, B^{i}\right)$ and moreover, $h_{i}^{2} \geq 1,(i=1,2)$. If we can take $(d, b)^{ \pm}$satisfying (2.10), (2.11), (2.16), (2.17), such that

$$
\left.\frac{P_{i}^{-}+\zeta^{-}}{h_{i}^{-}}=\frac{P_{j}^{+}+\zeta^{+}}{h_{j}^{+}} \quad \text { (for some } i, j=1,2\right)
$$

then we would make $s$ this number, and the Rankine-Hugoniot condition is satisfied with $\zeta^{ \pm} \neq \pm 1$. In fact, we observe that the above equality is equation (2.5), and we can make three different choices: $i=j=1, i=j=2$, and $i=1, j=2$. By symmetry, $i=1, j=2$ and $i=2, j=1$ are equivalent.

Again, we suppose $\left|(d, b)^{ \pm}\right| \ll 1$. Hence, the conditions (2.16), (2.17) are trivially satisfied. Furthermore, the function $\phi\left(\zeta^{+}, \zeta^{-}\right)$with $\zeta^{+}, \zeta^{-}$given respectively by (2.13), (2.14), where by abuse of notation we shall write $\phi\left((d, b)^{ \pm}\right)$, could be approximated in a suitable manner. Since $h_{1}^{ \pm} \sim 1, h_{2}^{ \pm} \sim\left|p^{ \pm}\right|^{-1}$, it follows that

$$
\frac{P_{1}^{ \pm}}{h_{1}^{ \pm}} \sim p^{ \pm} \quad \text { and } \quad \frac{P_{2}^{ \pm}}{h_{2}^{ \pm}} \sim \operatorname{sgn}\left(p^{ \pm}\right)
$$

Thus, considering (2.18), we have the following approximations:

$$
\begin{aligned}
\tilde{\phi}_{11}\left((d, b)^{ \pm}\right)= & p^{+}-p^{-}+2 \frac{\left(\gamma^{+}-\gamma^{-}\right)\left(\alpha^{+}-\alpha^{-}\right)}{\alpha^{+} \gamma^{-}-\alpha^{-} \gamma^{+}}, \\
\tilde{\phi}_{12}\left((d, b)^{ \pm}\right)= & p^{+}-p^{-}+2 \frac{\left(\gamma^{+}-\gamma^{-}\right)\left(\alpha^{+}-p^{-} \alpha^{-}\right)}{\alpha^{+} \gamma^{-}-\alpha^{-} \gamma^{+}}, \\
\tilde{\phi}_{22}\left((d, b)^{ \pm}\right)= & \operatorname{sgn}\left(p^{+}\right)-\operatorname{sgn}\left(p^{-}\right) \\
& +\frac{\left(\gamma^{+}-\gamma^{-}\right)\left(\alpha^{+}\left|p^{+}\right|-\alpha^{-}\left|p^{-}\right|\right)+\left(\alpha^{+}-\alpha^{-}\right)\left(\gamma^{+}\left|p^{+}\right|-\gamma^{-}\left|p^{-}\right|\right)}{\alpha^{+} \gamma^{-}-\alpha^{-} \gamma^{+}},
\end{aligned}
$$

where for $\tilde{\phi}_{12}$ we assumed, without loss of generality, that $p^{-}>0$. If one of the above functions is null, in a non-trivial manner, i.e., $\left|(d, b)^{ \pm}\right|>0$, over the manifold $\mathcal{M}$, then we have a non-trivial, small solution of (2.5). Set $\tilde{\phi}_{11}=0$; then

$$
\left\{\left(p^{+}-p^{-}\right)\left(\alpha^{+} \gamma^{-}-\alpha^{-} \gamma^{+}\right)\right\}+2\left\{\left(\gamma^{+}-\gamma^{-}\right)\left(\alpha^{+}-\alpha^{-}\right)\right\}=0 .
$$

The first parenthesis is of $O\left(\left|d_{i}^{ \pm} d_{j}^{ \pm} b_{i}^{ \pm} b_{j}^{ \pm}\right|\right)$, and the second is of $O\left(\left|d_{i}^{ \pm} d_{j}^{ \pm}\right|+\left|d_{i}^{ \pm} b_{j}^{ \pm}\right|+\right.$ $\left.\left|b_{i}^{ \pm} b_{j}^{ \pm}\right|\right)$for $(i, j=2,3)$. Then, it is not possible to obtain $\tilde{\phi}_{11}=0$, since we must have

$$
\gamma^{+}=\gamma^{-} \quad \text { or } \quad \alpha^{+}=\alpha^{-} \text {. }
$$

In fact, we already know that $\tilde{\phi}_{11}$ is not relevant. Since $\left|(d, b)^{ \pm}\right| \ll 1$ and $h^{ \pm} \sim 1$, it follows that $\left|(D, B)^{ \pm}\right| \ll 1$. Hence, from Remark 2.1 the jump discontinuity must be a contact discontinuity. Now, we set $\tilde{\phi}_{12}=0$; that is

$$
\left\{\left(p^{+}-p^{-}\right)\left(\alpha^{+} \gamma^{-}-\alpha^{-} \gamma^{+}\right)-2 p^{-} \alpha^{-}\left(\gamma^{+}-\gamma^{-}\right)\right\}+2\left\{\left(\gamma^{+}-\gamma^{-}\right) \alpha^{+}\right\}=0 .
$$


Again, the first parenthesis is of $O\left(\left|d_{i}^{ \pm} d_{j}^{ \pm} b_{i}^{ \pm} b_{j}^{ \pm}\right|\right)$, and the second is of $O\left(\left|d_{i}^{ \pm} d_{j}^{ \pm}\right|+\left|d_{i}^{ \pm} b_{j}^{ \pm}\right|+\right.$ $\left.\left|b_{i}^{ \pm} b_{j}^{ \pm}\right|\right)$for $(i, j=2,3)$. Then, it is not possible to obtain $\tilde{\phi}_{12}=0$, since we must have

$$
\gamma^{+}=\gamma^{-} \text {or } \alpha^{+}=0 .
$$

The condition $\alpha^{+}=0$ could be acceptable as we have observed, but this implies a contradiction with the assumption that $p^{-}>0$. Indeed, if $\alpha^{+}=0$, then we must have

$$
\left(d_{2}^{-}+b_{3}^{-}\right)=0 \text { and }\left(d_{3}^{-}-b_{2}^{-}\right)=0,
$$

which imply

$$
p^{-}=-\left|d^{-}\right|^{2}=-\left|b^{-}\right|^{2}<0 .
$$

Finally, we set $\tilde{\phi}_{22}=0$; then

$$
\begin{aligned}
& {\left[\operatorname{sgn}\left(p^{+}\right)-\operatorname{sgn}\left(p^{-}\right)\right]\left(\alpha^{+} \gamma^{-}-\alpha^{-} \gamma^{+}\right)} \\
& +\left(\gamma^{+}-\gamma^{-}\right)\left(\alpha^{+}\left|p^{+}\right|-\alpha^{-}\left|p^{-}\right|\right)+\left(\alpha^{+}-\alpha^{-}\right)\left(\gamma^{+}\left|p^{+}\right|-\gamma^{-}\left|p^{-}\right|\right)=0 .
\end{aligned}
$$

Now, if we prove that

$$
\left(2 \alpha^{+} \gamma^{+}-\alpha^{+} \gamma^{-}-\alpha^{-} \gamma^{+}\right) p^{+}+\left(2 \alpha^{-} \gamma^{-}-\alpha^{-} \gamma^{+}-\alpha^{+} \gamma^{-}\right) p^{-}=0,
$$

and moreover we have

$$
\left(2 \alpha^{+} \gamma^{+}-\alpha^{+} \gamma^{-}-\alpha^{-} \gamma^{+}\right)\left(2 \alpha^{-} \gamma^{-}-\alpha^{-} \gamma^{+}-\alpha^{+} \gamma^{-}\right)<0,
$$

then we are done; that is, $p^{+} p^{-}>0$ and thus $\tilde{\phi}_{22}=0$. Conveniently, we write

$$
\begin{aligned}
& 4 p^{+}=R-S, \\
& 4 p^{-}=\left[\left(\alpha^{+} \gamma^{-}\right)^{2} R-\left(\alpha^{-} \gamma^{+}\right)^{2} S\right] /\left(\alpha^{-} \gamma^{-}\right)^{2},
\end{aligned}
$$

where we have used (2.6), (2.7), and

$$
\begin{aligned}
& R=\left[\left(d_{2}^{+}+b_{3}^{+}\right)^{2}+\left(d_{3}^{+}-b_{2}^{+}\right)^{2}\right], \\
& S=\left[\left(d_{2}^{+}-b_{3}^{+}\right)^{2}+\left(d_{3}^{+}+b_{2}^{+}\right)^{2}\right] .
\end{aligned}
$$

From (2.19) and (2.21), we obtain

$$
\left(\alpha^{+} \gamma^{-}+\alpha^{-} \gamma^{+}\right)\left[\left(\gamma^{-}\right)^{2}\left(\alpha^{+}-\alpha^{-}\right)^{2} R-\left(\alpha^{-}\right)^{2}\left(\gamma^{+}-\gamma^{-}\right)^{2} S\right]=0 .
$$

Consequently, we must have

$$
\left(\alpha^{+} \gamma^{-}+\alpha^{-} \gamma^{+}\right)=0
$$

or

$$
\left(\gamma^{-}\right)^{2}\left(\alpha^{+}-\alpha^{-}\right)^{2} R=\left(\alpha^{-}\right)^{2}\left(\gamma^{+}-\gamma^{-}\right)^{2} S,
$$

and we observe that none of these imply a contradiction. Let $m, n \in \mathbb{R}^{*}, m \neq n$, such that

$$
\gamma^{+}=m \gamma^{-} \quad \text { and } \quad \alpha^{+}=n \alpha^{-} .
$$

It follows from (2.6) and (2.7) that

$$
\begin{aligned}
& \left(d_{2}^{-}-b_{3}^{-}\right)-m\left(d_{2}^{+}-b_{3}^{+}\right)=0, \\
& \left(d_{3}^{-}+b_{2}^{-}\right)-m\left(d_{3}^{+}+b_{2}^{+}\right)=0, \\
& \left(d_{2}^{-}+b_{3}^{-}\right)-n\left(d_{2}^{+}+b_{3}^{+}\right)=0, \\
& \left(d_{3}^{-}-b_{2}^{-}\right)-n\left(d_{3}^{+}-b_{2}^{+}\right)=0,
\end{aligned}
$$


which define a sub-manifold $\mathcal{N} \subset \mathcal{M}$ of dimension 4. Hence, for $(d, b)^{ \pm} \in \mathcal{N},(\underline{2.20})$ implies

$$
(2 n m-n-m)(2-m-n)<0 .
$$

We seek if there exist $m, n$ satisfying (2.25), which solves (2.22) or (2.23). The former, i.e., $\alpha^{-} \gamma^{+}=-\alpha^{+} \gamma^{-}$, implies $m=-n$, and thus condition (2.25) is trivially satisfied, that is,

$$
m=-n \Rightarrow(2 n m-n-m)(2-m-n)=-4 m^{2}<0 .
$$

Consequently, over $\mathcal{N}$ with $m=-n$, we have $\tilde{\phi}_{22}=0$. Furthermore, from (2.21) we get

$$
p^{-}=m^{2} p^{+},
$$

and from equations (2.13), (2.14) we obtain respectively

$$
\zeta^{+}=m \quad \text { and } \quad \zeta^{-}=1 / m .
$$

Therefore, we have two solutions:

$$
\zeta^{+}= \pm \sqrt{\frac{p^{-}}{p^{+}}}, \quad \zeta^{-}= \pm \sqrt{\frac{p^{+}}{p^{-}}},
$$

which yield a difference of \pm 1 when $p^{+} \neq p^{-}$. Moreover, for $u^{-}$sufficiently close to $u^{+}$, and thus from (1.4) $h^{-} \sim h^{+}$and $P^{-} \sim P^{+}$, we have $\zeta^{ \pm} \sim \pm 1$. Therefore, we recover the contact discontinuity solution in the limit case of weak jump discontinuities. The second condition, i.e., 2.23), implies

$$
(n-1)^{2} R=(m-1)^{2} S,
$$

i.e., one equation for two variables, which means that we have many solutions for $m, n$ and one of them could satisfy (2.25). Although, for $u^{-}$sufficiently close to $u^{+}$, from (2.21) we have

$$
\left(1-n^{2}\right) R=\left(1-m^{2}\right) S .
$$

Then, from (2.27) and (2.28) we must have $m=n$, which is a contradiction. Therefore, for all $m, n$ satisfying (2.27), we do not recover the contact discontinuity solution. Consequently, from now on, we consider $m=-n$. We remember that we have supposed $p^{+} p^{-} \neq 0$. From (2.21),

$$
p^{+}=0 \Leftrightarrow p^{-}=0,
$$

which implies $\zeta^{ \pm}= \pm 1$. Consequently, for $\zeta^{ \pm} \neq \pm 1$, we have $p^{+} p^{-}>0$.

In fact, we have shown that $\tilde{\phi}_{22}=0$, but not $\phi$ yet. Before we do this, we shall show some qualitative properties of the jump discontinuities given by (2.26). Let $\zeta^{+}, \zeta^{-}$be given by 2.26); when $p^{-}>p^{+}$, we have

$$
\begin{array}{cc}
\zeta^{+}<-1 \Leftrightarrow \zeta^{-}>-1 & \left(\zeta^{ \pm}<0\right), \\
\zeta^{+}>1 \Leftrightarrow \zeta^{-}<1 & \left(\zeta^{ \pm}>0\right) .
\end{array}
$$


In the first case, we obtain

$$
\begin{aligned}
& \lambda^{-}\left(u^{-}\right)=\frac{P^{-}-1}{h^{-}}<\frac{P^{-}+\zeta^{-}}{h^{-}}=s, \\
& \lambda^{+}\left(u^{-}\right)=\frac{P^{-}+1}{h^{-}}>\frac{P^{-}+\zeta^{-}}{h^{-}}=s, \\
& \lambda^{-}\left(u^{+}\right)=\frac{P^{+}-1}{h^{+}}>\frac{P^{+}+\zeta^{+}}{h^{+}}=s .
\end{aligned}
$$

Consequently, from Definition 1.3 we have a Rarefaction Shock, since

$$
\begin{array}{r}
\lambda^{-}\left(u^{-}\right)<s<\lambda^{-}\left(u^{+}\right), \\
s<\lambda^{+}\left(u^{-}\right) .
\end{array}
$$

In the second case, we obtain

$$
\begin{aligned}
& \lambda^{+}\left(u^{-}\right)=\frac{P^{-}+1}{h^{-}}>\frac{P^{-}+\zeta^{-}}{h^{-}}=s, \\
& \lambda^{-}\left(u^{-}\right)=\frac{P^{-}-1}{h^{-}}<\frac{P^{-}+\zeta^{-}}{h^{-}}=s, \\
& \lambda^{+}\left(u^{+}\right)=\frac{P^{+}+1}{h^{+}}<\frac{P^{+}+\zeta^{+}}{h^{+}}=s .
\end{aligned}
$$

Consequently, from Definition 1.3 we have a Lax Shock, since

$$
\begin{aligned}
& \lambda^{+}\left(u^{+}\right)<s<\lambda^{+}\left(u^{-}\right), \\
& \lambda^{-}\left(u^{-}\right)<s .
\end{aligned}
$$

Analogously, when $p^{-}<p^{+}$, we have

$$
\begin{array}{cc}
\zeta^{+}>-1 \Leftrightarrow \zeta^{-}<-1 & \left(\zeta^{ \pm}<0\right), \\
\zeta^{+}<1 \Leftrightarrow \zeta^{-}>1 & \left(\zeta^{ \pm}>0\right) .
\end{array}
$$

From the former, we obtain

$$
\begin{aligned}
& \lambda^{-}\left(u^{-}\right)=\frac{P^{-}-1}{h^{-}}>\frac{P^{-}+\zeta^{-}}{h^{-}}=s, \\
& \lambda^{-}\left(u^{+}\right)=\frac{P^{+}-1}{h^{+}}<\frac{P^{+}+\zeta^{+}}{h^{+}}=s, \\
& \lambda^{+}\left(u^{+}\right)=\frac{P^{+}+1}{h^{+}}>\frac{P^{+}+\zeta^{+}}{h^{+}}=s .
\end{aligned}
$$

Consequently, from Definition 1.3 we have a Lax Shock, since

$$
\begin{array}{r}
\lambda^{-}\left(u^{+}\right)<s<\lambda^{-}\left(u^{-}\right), \\
s<\lambda^{+}\left(u^{+}\right) .
\end{array}
$$


For the second, we obtain

$$
\begin{aligned}
& \lambda^{+}\left(u^{-}\right)=\frac{P^{-}+1}{h^{-}}<\frac{P^{-}+\zeta^{-}}{h^{-}}=s, \\
& \lambda^{-}\left(u^{+}\right)=\frac{P^{+}-1}{h^{+}}<\frac{P^{+}+\zeta^{+}}{h^{+}}=s, \\
& \lambda^{+}\left(u^{+}\right)=\frac{P^{+}+1}{h^{+}}>\frac{P^{+}+\zeta^{+}}{h^{+}}=s .
\end{aligned}
$$

Consequently, from Definition 1.3 we have a Rarefaction Shock, since

$$
\begin{aligned}
& \lambda^{+}\left(u^{-}\right)<s<\lambda^{+}\left(u^{+}\right), \\
& \lambda^{-}\left(u^{+}\right)<s .
\end{aligned}
$$

Then it remains to show that $\phi=0$. The following lemma will be used in the proof of it.

Lemma 2.3. Let $\mathcal{U}$ be the manifold defined as

$$
\mathcal{U}:=\left\{A \in M_{n}(\mathbb{R}): \operatorname{det} A=0,\|A\|=1\right\} .
$$

Then $\mathcal{U}$ is a connected set.

Proof. 1. Let $a \in \mathbb{R}^{n},\|a\|=1$, and we set

$$
\mathcal{U}(a):=\left\{A \in M_{n}(\mathbb{R}) ; A a=0,\|A\|=1\right\} .
$$

Then, we have

Furthermore, we define

$$
\mathcal{U}=\bigcup_{\|a\|=1} \mathcal{U}(a)
$$

$$
\mathcal{U}_{1}:=\left\{A \in M_{n}(\mathbb{R}) ; A^{T} e_{1}=0\right\},
$$

where $\left\{e_{i}\right\}_{i=1}^{n}$ is the standard basis of $\mathbb{R}^{n}$. Then,

$$
\mathcal{U}(a) \cap \mathcal{U}_{1} \neq \emptyset,
$$

for any vector $a$. Indeed, if we take $M=e_{2} \otimes b$, where $b \in \mathbb{R}^{n},\|b\|=1$, and $b \cdot a=0$, then $\|M\|=\left\|e_{2}\right\|\|b\|=1$, and

$$
\begin{aligned}
& M a=\left(e_{2} \otimes b\right) a=(b \cdot a) e_{2}=0, \\
& M^{T} e_{1}=\left(e_{2} \otimes b\right)^{T} e_{1}=\left(b \otimes e_{2}\right) e_{1}=\left(e_{2} \cdot e_{1}\right) b=0,
\end{aligned}
$$

that is, $M \in \mathcal{U}(a) \cap \mathcal{U}_{1}$.

2. Now, we observe that $\mathcal{U}(a)$ is equivalent to the unit sphere of $M_{n \times(n-1)}(\mathbb{R})$, and thus pathwise-connected. Indeed, upon rotating and relabelling the coordinate axes if necessary, we may assume $a=e_{1}$. Therefore,

$$
\begin{aligned}
\mathcal{U}(a) \sim \mathcal{U}\left(e_{1}\right)=\left\{A \in M_{n}(\mathbb{R}) ; A_{i 1}=\right. & 0, A_{i(j+1)}=B_{i j}, \\
& \left.B \in M_{n \times(n-1)}(\mathbb{R}),\|B\|=1\right\} .
\end{aligned}
$$

It follows easily that $\mathcal{U}\left(e_{1}\right)$ is pathwise-connected. Analogously, we have that $\mathcal{U}_{1}$ is pathwise-connected. 
3. Finally, let $A_{1}, A_{2} \in \mathcal{U}$. Then, there exist $a_{1}, a_{2} \in \mathbb{R}^{n},\left\|a_{1}\right\|=\left\|a_{2}\right\|=1$, such that

$$
A_{1} \in \mathcal{U}\left(a_{1}\right), \quad A_{2} \in \mathcal{U}\left(a_{2}\right) .
$$

Since $\mathcal{U}_{1}, \mathcal{U}\left(a_{1}\right)$, and $\mathcal{U}\left(a_{2}\right)$ are pathwise-connected and $\mathcal{U}(a) \cap \mathcal{U}_{1} \neq \emptyset$ for any $a$, we can always make a path in $\mathcal{U}$ with endpoints $A_{1}, A_{2}$. Therefore, $\mathcal{U}$ is pathwise-connected, and thus a connected set.

Theorem 2.1. Let (1.8)-(1.11) be the system of Born-Infeld equations, and let us now consider the Riemann Problem. Then there exist large states

$$
u^{-}=\left(D_{2}^{-}, D_{3}^{-}, B_{2}^{-}, B_{3}^{-}\right), \quad u^{+}=\left(D_{2}^{+}, D_{3}^{+}, B_{2}^{+}, B_{3}^{+}\right)
$$

that can be connected by jump discontinuities, which are not contact discontinuities. Moreover, these jump discontinuities could be either Lax Shocks or Rarefaction Shocks.

Proof. 1. Let $\mathcal{M}$ be the manifold defined by (2.10), 2.11). Since $\mathcal{M}$ is conic, for all $0 \neq \omega \in \mathcal{M}$, we may write $\omega=(\mu, v)$ with $\mu \in \mathbb{R}^{+}$and $v \in \mathcal{V}$, that is

$$
\mathcal{M}=(0, \infty) \times \mathcal{V},
$$

where $\mathcal{V}$ is a manifold of dimension 5 . Moreover, we may assume without loss of generality that

$$
\mathcal{V}=\mathcal{M} \cap S^{7}
$$

and thus $\|\omega\|=\mu$. Further, $\mathcal{V}$ is globally compact. Analogously, for $\mathcal{N}$, the manifold defined by (2.24) with $m=-n$, we have

$$
\mathcal{N}=(0, \infty) \times \mathcal{W},
$$

where $\mathcal{W}$ is a manifold of dimension 3. As $\mathcal{N} \subset \mathcal{M}$, it follows that $\mathcal{W} \subset \mathcal{V}$.

2. For $\alpha^{+} \gamma^{-}-\alpha^{-} \gamma^{+} \neq 0, \phi: \mathcal{M} \rightarrow \mathbb{R}$, given by (2.5), (2.13), and (2.14), is well defined and, moreover, a $C^{\infty}(\mathcal{M})$-function. Let $\delta>0$ be sufficiently small. We set $\tilde{\phi}: \mathcal{M} \rightarrow \mathbb{R}$,

$$
\tilde{\phi}(\mu, v)=\tilde{\phi}_{22}(\mu, v)
$$

for any $\mu<\delta$ and $v \in \mathcal{V}$. Since $\tilde{\phi}$ is not a continuous function, for each $\ell \in \mathbb{Z}^{+}$, we define $\tilde{\phi}_{\ell}: \mathcal{M} \rightarrow \mathbb{R}$

$$
\begin{aligned}
\tilde{\phi}_{\ell}(\mu, v) & :=H_{\ell}^{\prime}\left(p^{+}\right)-H_{\ell}^{\prime}\left(p^{-}\right) \\
& +\frac{\left(\gamma^{+}-\gamma^{-}\right)\left(\alpha^{+} H_{\ell}\left(p^{+}\right)-\alpha^{-} H_{\ell}\left(p^{-}\right)\right)+\left(\alpha^{+}-\alpha^{-}\right)\left(\gamma^{+} H_{\ell}\left(p^{+}\right)-\gamma^{-} H_{\ell}\left(p^{-}\right)\right)}{\alpha^{+} \gamma^{-}-\alpha^{-} \gamma^{+}}
\end{aligned}
$$

for all $\mu<\delta$ and $v \in \mathcal{V}$, where

$$
H_{\ell}(z)=\left(z^{2}+1 / \ell^{2}\right)^{1 / 2}-1 / \ell \quad\left(z \in \mathbb{R}, \ell \in \mathbb{Z}^{+}\right) .
$$

Consequently, $\tilde{\phi}_{\ell} \in C^{\infty}(\mathcal{M})$ and $\tilde{\phi}_{\ell} \rightarrow \tilde{\phi}$ uniformly as $\ell \rightarrow \infty$; that is, given $\varepsilon>0$, there exists $L \in \mathbb{Z}^{+}$such that, for any $\mu<\delta$ and $v \in \mathcal{V}$,

$$
\tilde{\phi}(\mu, v)-\varepsilon<\tilde{\phi}_{\ell}(\mu, v)<\tilde{\phi}(\mu, v)+\varepsilon,
$$

for all $\ell \geq L$. Furthermore, since $\tilde{\phi}(\mu, w)=0$ for all $(\mu, w) \in(0, \delta) \times \mathcal{W}$, and $\varepsilon>0$ is arbitrary, we have for any $\ell \geq L$

$$
\tilde{\phi}_{\ell}(\mu, w)=0 .
$$


Therefore, there exist $v_{1}, v_{2} \in \mathcal{V}$ such that, for all $\ell \geq L$,

$$
\tilde{\phi}_{\ell}\left(\mu, v_{1}\right)<0<\tilde{\phi}_{\ell}\left(\mu, v_{2}\right) \text {. }
$$

3. Finally, let us show that there exists $\left(\mu_{0}, v_{0}\right) \in \mathcal{M}$ such that $\phi\left(\mu_{0}, v_{0}\right)=0$. For $\delta>0$ above, we have $\phi(\mu, v) \sim \tilde{\phi}(\mu, v)$ for all $(\mu, v) \in(0, \delta) \times \mathcal{V}$; then for $\ell \in \mathbb{Z}^{+}$ sufficiently large, it follows that $\phi(\mu, v) \sim \tilde{\phi}_{\ell}(\mu, v)$. In fact, since $\phi, \tilde{\phi}_{\ell}$ are continuous functions and $\mathcal{V}$ is compact, given $\varepsilon>0$, there exist $0<\delta_{1}<\delta_{2}<\delta$ such that, for any $(\mu, v) \in\left[\delta_{1}, \delta_{2}\right] \times \mathcal{V}$,

$$
\phi(\mu, v)-\varepsilon<\tilde{\phi}_{\ell}(\mu, v)<\phi(\mu, v)+\varepsilon .
$$

Now, from item 2 there exist $v_{1}, v_{2} \in \mathcal{V}$ such that, for any $\mu \in\left[\delta_{1}, \delta_{2}\right]$,

$$
\phi\left(\mu, v_{1}\right)-\varepsilon<\tilde{\phi}_{\ell}\left(\mu, v_{1}\right)<0<\tilde{\phi}_{\ell}\left(\mu, v_{2}\right)<\phi\left(\mu, v_{2}\right)+\varepsilon .
$$

Letting $\varepsilon \rightarrow 0^{+}$, there exists $\mu_{0} \in\left[\delta_{1}, \delta_{2}\right]$ such that

$$
\phi\left(\mu_{0}, v_{1}\right)<0<\phi\left(\mu_{0}, v_{2}\right) .
$$

Thus from the Intermediate Value Theorem, there exists $v_{0} \in \mathcal{V}$ such that

$$
\phi\left(\mu_{0}, v_{0}\right)=0,
$$

where we have used that $\mathcal{V}$ is connected, which follows easy from Lemma 2.3. Indeed, it is enough to observe that $\mathcal{V}=\mathcal{U} \times \mathcal{U}$.

3. The ill-posed Riemann problem. Since shocks could be formed, we shall show in this section that a further microscopic, i.e., local, theory is needed to complete the Born-Infeld model. In fact, as the following theorem shows, this could happen in the presence of rarefaction shocks. In this case, the Riemann Problem for the system of Born-Infeld equations can have many solutions, and thus the Born-Infeld model is not complete by itself.

Theorem 3.1. Let $u^{\ell}=\left(D_{2}^{\ell}, D_{3}^{\ell}, B_{2}^{\ell}, B_{3}^{\ell}\right), u^{r}=\left(D_{2}^{r}, D_{3}^{r}, B_{2}^{r}, B_{3}^{r}\right)$ be two states satisfying the Rankine-Hugoniot condition for the system of Born-Infeld equations. If $u^{\ell}, u^{r}$ are connected by a rarefaction shock, then the Riemann Problem of (1.8)-(1.11) in $\mathbb{R}^{+} \times \mathbb{R}$, with initial-data

$$
\left(D_{2}, D_{3}, B_{2}, B_{3}\right)(0, x)= \begin{cases}\left(D_{2}^{\ell}, D_{3}^{\ell}, B_{2}^{\ell}, B_{3}^{\ell}\right) & \text { if } x<0, \\ \left(D_{2}^{r}, D_{3}^{r}, B_{2}^{r}, B_{3}^{r}\right) & \text { if } x>0,\end{cases}
$$

is not well-posed. Moreover, the Born-Infeld model is not complete.

Proof. 1. By hypothesis, the states $u^{\ell}, u^{r}$ satisfy the Rankine-Hugoniot jump condition of a rarefaction shock; we denote by $s$ its speed. So from Theorem 2.1,

$$
s=\frac{P^{\ell}+\zeta^{\ell}}{h^{\ell}}=\frac{P^{r}+\zeta^{r}}{h^{r}}
$$

with $\zeta^{\ell}, \zeta^{r} \neq \pm 1, P^{\ell} P^{r} \geq 0$, where $h$ and $P$ are given by (1.4) with the obvious notations. Let $\tilde{u}^{\ell}, \tilde{u}^{r}$ be two states to be determined, and we assume that $\left(\tilde{u}^{\ell}, \tilde{u}^{r}\right) \in \mathbb{R}^{8}$ is close enough to $\left(u^{\ell}, u^{r}\right) \in \mathbb{R}^{8}$; that is,

$$
u^{\ell} \sim \tilde{u}^{\ell} \quad \text { and } \quad \tilde{u}^{r} \sim u^{r} .
$$


Therefore, $u^{\ell}, \tilde{u}^{\ell}$ and $\tilde{u}^{r}, u^{r}$ could be connected by two different contact discontinuities. For instance, we set

$$
\begin{aligned}
& s_{1}=\lambda^{-}\left(u^{\ell}\right)=\lambda^{-}\left(\tilde{u}^{\ell}\right), \\
& s_{2}=\lambda^{+}\left(\tilde{u}^{r}\right)=\lambda^{+}\left(u^{r}\right),
\end{aligned}
$$

with $s_{1}<s_{2}$, the respective speeds of their contact discontinuities. Now, we seek if there exists a jump discontinuity such that $\tilde{u}^{\ell}, \tilde{u}^{r}$ are connected by it. Let us denote by $\tilde{s}$ its speed. Moreover, we must have

$$
s_{1}<\tilde{s}<s_{2},
$$

and this jump discontinuity must be a rarefaction shock. Indeed, it is not possible to have $\tilde{s}<s_{1}$ or $\tilde{s}>s_{2}$, since we have assumed $\left(\tilde{u}^{\ell}, \tilde{u}^{r}\right) \sim\left(u^{\ell}, u^{r}\right)$. Furthermore, we have

$$
s_{1}=\frac{\tilde{P}^{\ell}-1}{\tilde{h}^{\ell}}<\frac{\tilde{P}^{\ell}+\zeta^{-}}{\tilde{h}^{\ell}}=\tilde{s} \quad\left(\zeta^{-}>-1 \Leftrightarrow \zeta^{+}<-1\right),
$$

i.e., $\tilde{s}$ is a rarefaction shock. Analogously,

$$
s_{2}=\frac{\tilde{P}^{r}+1}{\tilde{h}^{r}}>\frac{\tilde{P}^{r}+\zeta^{+}}{\tilde{h}^{r}}=\tilde{s} \quad\left(\zeta^{+}<1 \Leftrightarrow \zeta^{-}>1\right) .
$$

2. The states $u^{\ell}, \tilde{u}^{\ell}, \tilde{u}^{r}$, and $u^{r}$ of item 1 have to satisfy the following Rankine-Hugoniot jump conditions:

$$
\begin{aligned}
& s_{1}\left(\tilde{D}_{2}^{\ell}-D_{2}^{\ell}\right)=\left(\tilde{B}_{3}^{\ell}+\tilde{D}_{2}^{\ell} \tilde{P}^{\ell}\right)\left(\tilde{h}^{\ell}\right)^{-1}-\left(B_{3}^{\ell}+D_{2}^{\ell} P^{\ell}\right)\left(h^{\ell}\right)^{-1}, \\
& s_{1}\left(\tilde{D}_{3}^{\ell}-D_{3}^{\ell}\right)=\left(-\tilde{B}_{2}^{\ell}+\tilde{D}_{3}^{\ell} \tilde{P}^{\ell}\right)\left(\tilde{h}^{\ell}\right)^{-1}-\left(-B_{2}^{\ell}+D_{3}^{\ell} P^{\ell}\right)\left(h^{\ell}\right)^{-1}, \\
& s_{1}\left(\tilde{B}_{2}^{\ell}-B_{2}^{\ell}\right)=\left(-\tilde{D}_{3}^{\ell}+\tilde{B}_{2}^{\ell} \tilde{P}^{\ell}\right)\left(\tilde{h}^{\ell}\right)^{-1}-\left(-D_{3}^{\ell}+B_{2}^{\ell} P^{\ell}\right)\left(h^{\ell}\right)^{-1}, \\
& s_{1}\left(\tilde{B}_{3}^{\ell}-B_{3}^{\ell}\right)=\left(\tilde{D}_{2}^{\ell}+\tilde{B}_{3}^{\ell} \tilde{P}^{\ell}\right)\left(\tilde{h}^{\ell}\right)^{-1}-\left(D_{2}^{\ell}+B_{3}^{\ell} P^{\ell}\right)\left(h^{\ell}\right)^{-1}, \\
& \tilde{s}_{2}\left(\tilde{D}_{2}^{r}-\tilde{D}_{2}^{\ell}\right)=\left(\tilde{B}_{3}^{r}+\tilde{D}_{2}^{r} \tilde{P}^{r}\right)\left(\tilde{h}^{r}\right)^{-1}-\left(\tilde{B}_{3}^{\ell}+\tilde{D}_{2}^{\ell} \tilde{P}^{\ell}\right)\left(\tilde{h}^{\ell}\right)^{-1}, \\
& \tilde{s}\left(\tilde{D}_{3}^{r}-\tilde{D}_{3}^{\ell}\right)=\left(-\tilde{B}_{2}^{r}+\tilde{D}_{3}^{r} \tilde{P}^{r}\right)\left(\tilde{h}^{r}\right)^{-1}-\left(-\tilde{B}_{2}^{\ell}+\tilde{D}_{3}^{\ell} \tilde{P}^{\ell}\right)\left(\tilde{h}^{\ell}\right)^{-1}, \\
& \tilde{s}_{(}\left(\tilde{B}_{2}^{r}-\tilde{B}_{2}^{\ell}\right)=\left(-\tilde{D}_{3}^{r}+\tilde{B}_{2}^{r} \tilde{P}^{r}\right)\left(\tilde{h}^{r}\right)^{-1}-\left(-\tilde{D}_{3}^{\ell}+\tilde{B}_{2}^{\ell} \tilde{P}^{\ell}\right)\left(\tilde{h}^{\ell}\right)^{-1}, \\
& \tilde{s}_{3}\left(\tilde{B}_{3}^{r}-\tilde{B}_{3}^{\ell}\right)=\left(\tilde{D}_{2}^{r}+\tilde{B}_{3}^{r} \tilde{P}^{r}\right)\left(\tilde{h}^{r}\right)^{-1}-\left(\tilde{D}_{2}^{\ell}+\tilde{B}_{3}^{\ell} \tilde{P}^{\ell}\right)\left(\tilde{h}^{\ell}\right)^{-1}, \\
& s_{2}\left(D_{2}^{r}-\tilde{D}_{2}^{r}\right)=\left(B_{3}^{r}+D_{2}^{r} P^{r}\right)\left(h^{r}\right)^{-1}-\left(\tilde{B}_{3}^{r}+\tilde{D}_{2}^{r} \tilde{P}^{r}\right)\left(\tilde{h}^{r}\right)^{-1}, \\
& s_{2}\left(D_{3}^{r}-\tilde{D}_{3}^{r}\right)=\left(-B_{2}^{r}+D_{3}^{r} P^{r}\right)\left(h^{r}\right)^{-1}-\left(-\tilde{B}_{2}^{r}+\tilde{D}_{3}^{r} \tilde{P}^{r}\right)\left(\tilde{h}^{r}\right)^{-1}, \\
& s_{2}\left(B_{2}^{r}-\tilde{B}_{2}^{r}\right)=\left(-D_{3}^{r}+B_{2}^{r} P^{r}\right)\left(h^{r}\right)^{-1}-\left(-\tilde{D}_{3}^{r}+\tilde{B}_{2}^{r} \tilde{P}^{r}\right)\left(\tilde{h}^{r}\right)^{-1}, \\
& s_{2}\left(B_{3}^{r}-\tilde{B}_{3}^{r}\right)=\left(D_{2}^{r}+B_{3}^{r} P^{r}\right)\left(h^{r}\right)^{-1}-\left(\tilde{D}_{2}^{r}+\tilde{B}_{3}^{r} \tilde{P}^{r}\right)\left(\tilde{h}^{r}\right)^{-1} .
\end{aligned}
$$


From the above equations and $s_{1}, s_{2}$ given by (3.2), we obtain

$$
\begin{aligned}
& \psi_{1}:=\left[\tilde{D}_{2}^{\ell}+\tilde{B}_{3}^{\ell}\right]\left(\tilde{h}^{\ell}\right)^{-1}-\left[D_{2}^{\ell}+B_{3}^{\ell}\right]\left(h^{\ell}\right)^{-1}=0, \\
& \psi_{2}:=\left[\tilde{D}_{3}^{\ell}-\tilde{B}_{2}^{\ell}\right]\left(\tilde{h}^{\ell}\right)^{-1}-\left[D_{3}^{\ell}-B_{2}^{\ell}\right]\left(h^{\ell}\right)^{-1}=0, \\
& \psi_{3}:=\left[\tilde{B}_{3}^{r}-\tilde{D}_{2}^{r}\left(\tilde{s} \tilde{h}^{r}-\tilde{P}^{r}\right)\right]\left(\tilde{h}^{r}\right)^{-1}-\left[\tilde{B}_{3}^{\ell}-\tilde{D}_{2}^{\ell}\left(\tilde{s} \tilde{h}^{\ell}-\tilde{P}^{\ell}\right)\right]\left(\tilde{h}^{\ell}\right)^{-1}=0, \\
& \psi_{4}:=\left[-\tilde{B}_{2}^{r}-\tilde{D}_{3}^{r}\left(\tilde{s} \tilde{h}^{r}-\tilde{P}^{r}\right)\right]\left(\tilde{h}^{r}\right)^{-1}-\left[-\tilde{B}_{2}^{\ell}-\tilde{D}_{3}^{\ell}\left(\tilde{s} \tilde{h}^{\ell}-\tilde{P}^{\ell}\right)\right]\left(\tilde{h}^{\ell}\right)^{-1}=0, \\
& \psi_{5}:=\left[-\tilde{D}_{3}^{r}-\tilde{B}_{2}^{r}\left(\tilde{s} \tilde{h}^{r}-\tilde{P}^{r}\right)\right]\left(\tilde{h}^{r}\right)^{-1}-\left[-\tilde{D}_{3}^{\ell}-\tilde{B}_{2}^{\ell}\left(\tilde{s} \tilde{h}^{\ell}-\tilde{P}^{\ell}\right)\right]\left(\tilde{h}^{\ell}\right)^{-1}=0, \\
& \psi_{6}:=\left[\tilde{D}_{2}^{r}-\tilde{B}_{3}^{r}\left(\tilde{s} \tilde{h}^{r}-\tilde{P}^{r}\right)\right]\left(\tilde{h}^{r}\right)^{-1}-\left[\tilde{D}_{2}^{\ell}-\tilde{B}_{3}^{\ell}\left(\tilde{s} \tilde{h}^{\ell}-\tilde{P}^{\ell}\right)\right]\left(\tilde{h}^{\ell}\right)^{-1}=0, \\
& \psi_{7}:=\left[D_{2}^{r}-B_{3}^{r}\right]\left(h^{r}\right)^{-1}-\left[\tilde{D}_{2}^{r}-\tilde{B}_{3}^{r}\right]\left(\tilde{h}^{r}\right)^{-1}=0, \\
& \psi_{8}:=\left[D_{3}^{r}+B_{2}^{r}\right]\left(h^{r}\right)^{-1}-\left[\tilde{D}_{3}^{r}+\tilde{B}_{2}^{r}\right]\left(\tilde{h}^{r}\right)^{-1}=0 .
\end{aligned}
$$

Hence, we have 8 equations to be satisfied by 9 variables, that is, $\psi_{i}\left(\tilde{s}, \tilde{u}^{\ell}, \tilde{u}^{r}\right)=0$, $(i=1, \ldots, 8)$. If there exists $\left(s_{0}, u_{0}^{\ell}, u_{0}^{r}\right)$ such that

$$
\psi\left(s_{0}, u_{0}^{\ell}, u_{0}^{r}\right)=0
$$

then we have a solution of the Riemann problem.

3. By definition, $\psi: \mathbb{R} \times \mathbb{R}^{8} \rightarrow \mathbb{R}^{8}$ is a $C^{\infty}$ function; we set

$$
[A]_{i j}:=\partial_{(j+1)} \psi_{i}\left(s, u^{\ell}, u^{r}\right) \quad(i, j=1, \ldots, 8) .
$$


After a straightforward calculation, we obtain the matrix $A$; for convenience we write $A=B+C$, with $B$ and $C$ given respectively by

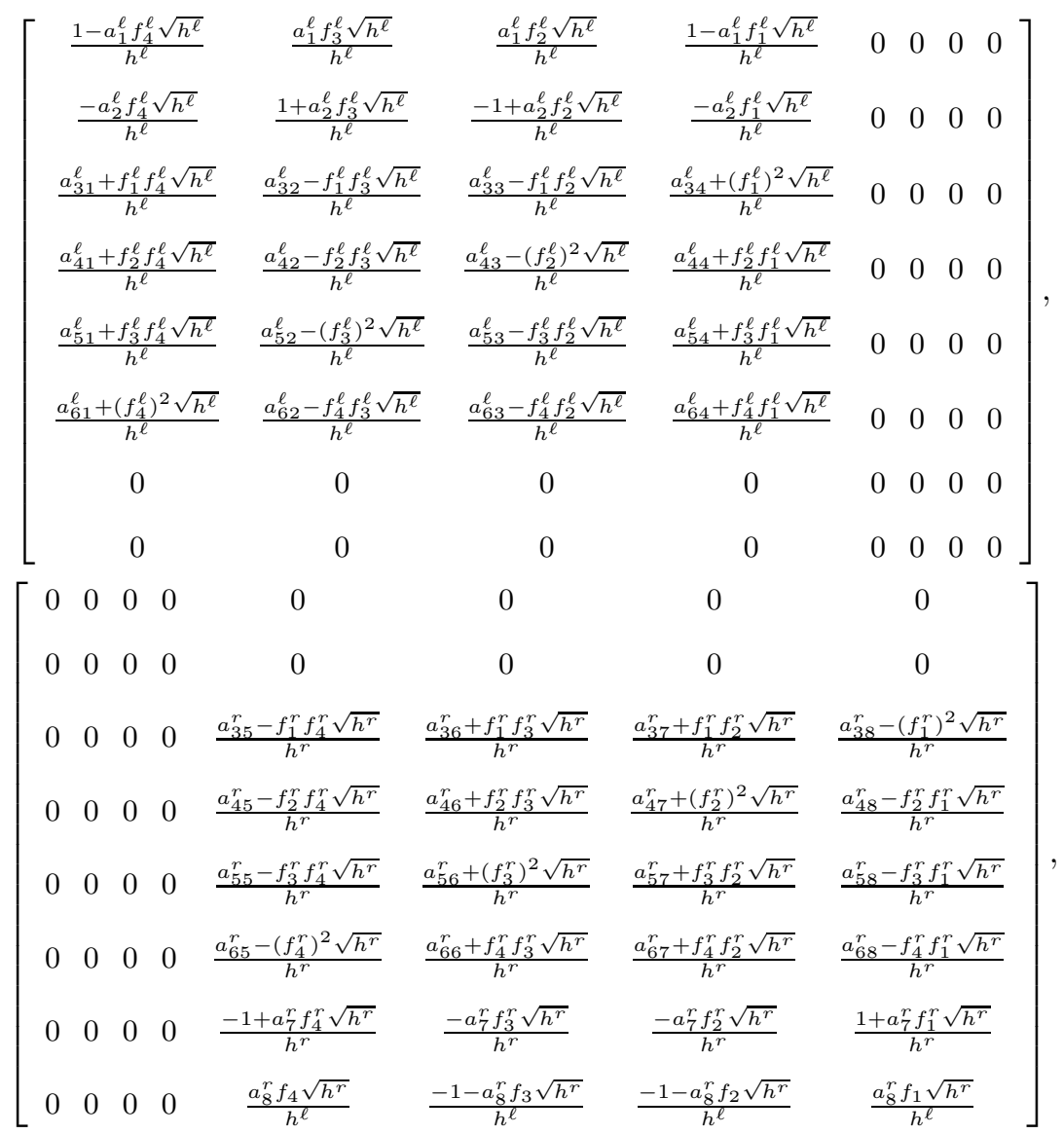

where $f^{\ell}=f\left(u^{\ell}\right), f^{r}=f\left(u^{r}\right), f$ is the flux function of the Born-Infeld system, and

$$
a_{1}^{\ell}=\frac{D_{2}^{\ell}+B_{3}^{\ell}}{h^{\ell}}, \quad a_{2}^{\ell}=\frac{D_{3}^{\ell}-B_{2}^{\ell}}{h^{\ell}}, \quad a_{7}^{r}=\frac{D_{2}^{r}-B_{3}^{r}}{h^{r}}, \quad a_{8}^{r}=\frac{D_{3}^{r}+B_{2}^{r}}{h^{r}} .
$$

Moreover, we have with the correspondent $\ell, r$ superscripts

$$
\begin{aligned}
& a_{31}=a_{64}=-a_{35}=-a_{68}=s h-\left(D_{2} B_{3}+P\right), \\
& a_{42}=a_{53}=-a_{46}=-a_{57}=s h-\left(-D_{3} B_{2}+P\right), \\
& a_{34}=-a_{38}=-\left[1+\left(D_{2}\right)^{2}\right], \quad a_{43}=-a_{47}=\left[1+\left(D_{3}\right)^{2}\right], \\
& a_{52}=-a_{56}=\left[1+\left(B_{2}\right)^{2}\right], \quad a_{61}=-a_{65}=-\left[1+\left(B_{3}\right)^{2}\right], \\
& a_{32}=-a_{54}=-a_{36}=a_{58}=D_{2} B_{2}, \quad a_{51}=-a_{62}=-a_{55}=a_{66}=-B_{2} B_{3}, \\
& a_{41}=-a_{63}=-a_{45}=a_{67}=-B_{3} D_{3}, \quad a_{33}=-a_{44}=-a_{37}=a_{48}=D_{3} D_{2} .
\end{aligned}
$$


Now, we study whether $A$ is non-singular. To do this, we verify whether some row or column is a linear combination of the others. We begin regarding the columns and, in this case from the particular structure of $A$, it is enough to verify for $B$ or $C$. Let us study $B$, and in order to simplify the notation, we drop the superscript $\ell$. Then, making a linear combination with the columns of $B$, we obtain

$$
\begin{array}{cc}
\alpha_{1} f_{4}-\alpha_{2} f_{3}-\alpha_{3} f_{2}+\alpha_{4} f_{1}=0, \\
\alpha_{1}+\alpha_{4}=0, & \alpha_{2}-\alpha_{3}=0 . \\
\sum_{i=1}^{4} \alpha_{i} a_{j i}=0 & (j=3,4,5,6) .
\end{array}
$$

The former equation of (3.4) implies for all $\left(D_{2}, D_{3}, B_{2}, B_{3}\right) \neq 0$,

$$
\begin{array}{ll}
\left(\alpha_{1}+\alpha_{4}\right)(P+1)=0, & \left(\alpha_{2}-\alpha_{3}\right)(P+1)=0 \\
\left(\alpha_{1}-\alpha_{4}\right)(P-1)=0, & \left(\alpha_{2}+\alpha_{3}\right)(P-1)=0 .
\end{array}
$$

Hence, when $P \neq \pm 1$, we have $\alpha_{i}=0,(i=1, \ldots, 4)$. However, we shall show that the columns of $A$ are linear independent in any case. Indeed, for $P=-1$, we have $\alpha_{1}=\alpha_{4}$ and $\alpha_{2}=-\alpha_{3}$. Then, from the second equation of (3.4),

$$
\alpha_{i}=0 \quad(i=1, \ldots, 4) .
$$

For $P=1$, we have $\alpha_{1}=-\alpha_{4}=: \alpha$ and $\alpha_{2}=\alpha_{3}=: \hat{\alpha}$; thus, the second equation of (3.4) is satisfied, and from (3.5),

$$
\begin{aligned}
& \alpha\left[s h+D_{2}\left(D_{2}-B_{3}\right)\right]+\hat{\alpha} D_{2}\left[B_{2}+D_{3}\right]=0, \\
& \alpha\left[s h-B_{3}\left(D_{2}-B_{3}\right)\right]-\hat{\alpha} B_{3}\left[B_{2}+D_{3}\right]=0, \\
& \alpha D_{3}\left[D_{2}-B_{3}\right]+\hat{\alpha}\left[s h+D_{3}\left(B_{2}+D_{3}\right)\right]=0, \\
& \alpha B_{2}\left[D_{2}-B_{3}\right]+\hat{\alpha}\left[s h+B_{2}\left(B_{2}+D_{3}\right)\right]=0 .
\end{aligned}
$$

That is, we have four linear homogeneous equations for the two unknowns $\alpha$ and $\hat{\alpha}$. It follows that we could get a solution $(\alpha, \hat{\alpha}) \neq 0$ if and only if $D_{2}=-B_{3}, D_{3}=B_{2}$ or $D_{2}=B_{3}, D_{3}=-B_{2}$, but both of them imply contradictions. In the first case, we have

$$
P=-|D|^{2}=-|B|^{2}<0
$$

For the second, we obtain

$$
\alpha s h=0, \quad \tilde{\alpha} s h=0,
$$

and since $h>0, s$ must be zero, which implies from item $1 \zeta=-1$. Consequently, the columns of $A$ are linear independent. Now, making a linear combination with the rows 
of $A$, we obtain

$$
\begin{gathered}
\alpha_{1} a_{1}^{\ell}+\alpha_{2} a_{2}^{\ell}-\sum_{i=3}^{6} \alpha_{i} f_{(i-2)}^{\ell}=0, \\
\sum_{i=3}^{6} \alpha_{i} f_{(i-2)}^{r}-\alpha_{7} a_{7}^{r}-\alpha_{8} a_{2}^{r}=0, \\
\alpha_{1}+\sum_{i=3}^{6} \alpha_{i} a_{i 1}^{\ell}=0, \quad \alpha_{2}+\sum_{i=3}^{6} \alpha_{i} a_{i 2}^{\ell}=0, \\
\alpha_{2}-\sum_{i=3}^{6} \alpha_{i} a_{i 3}^{\ell}=0, \quad \alpha_{1}+\sum_{i=3}^{6} \alpha_{i} a_{i 4}^{\ell}=0, \\
\sum_{i=3}^{6} \alpha_{i} a_{i 5}^{r}-\alpha_{7}^{r}=0, \quad \sum_{i=3}^{6} \alpha_{i} a_{i 6}^{r}-\alpha_{8}^{r}=0, \\
\sum_{i=3}^{6} \alpha_{i} a_{i 7}^{r}-\alpha_{8}^{r}=0, \quad \sum_{i=3}^{6} \alpha_{i} a_{i 8}^{r}+\alpha_{7}^{r}=0 .
\end{gathered}
$$

From (3.6), (3.7), we get for all $\left(D_{2}^{\ell}, D_{3}^{\ell}, B_{2}^{\ell}, B_{3}^{\ell}\right),\left(D_{2}^{r}, D_{3}^{r}, B_{2}^{r}, B_{3}^{r}\right) \neq 0$,

$$
\begin{array}{ll}
2 \alpha_{1}=\left(\alpha_{3}+\alpha_{6}\right)\left(P^{\ell}+1\right), & \left(\alpha_{3}-\alpha_{6}\right)\left(P^{\ell}-1\right)=0, \\
2 \alpha_{2}=\left(\alpha_{4}-\alpha_{5}\right)\left(P^{\ell}+1\right), & \left(\alpha_{4}+\alpha_{5}\right)\left(P^{\ell}-1\right)=0, \\
2 \alpha_{7}=\left(\alpha_{3}-\alpha_{6}\right)\left(P^{r}-1\right), & \left(\alpha_{3}+\alpha_{6}\right)\left(P^{r}+1\right)=0, \\
2 \alpha_{8}=\left(\alpha_{4}+\alpha_{5}\right)\left(P^{r}-1\right), & \left(\alpha_{4}-\alpha_{5}\right)\left(P^{r}+1\right)=0 .
\end{array}
$$

Then, when $P^{\ell}, P^{r} \neq \pm 1$, we have

$$
\alpha_{i}=0 \quad(i=1, \ldots, 8) .
$$

Moreover, since $P^{\ell} P^{r} \geq 0$, we obtain respectively for $P^{\ell}, P^{r}=-1$ and $P^{\ell}, P^{r}=1$

$$
\begin{aligned}
& \alpha_{1}=\alpha_{2}=\alpha_{7}=\alpha_{8}=0, \\
& \alpha_{3}=\alpha_{6}=\alpha, \alpha_{4}=-\alpha_{5}=\hat{\alpha}, \\
& \alpha_{1}=\alpha_{2}=\alpha_{7}=\alpha_{8}=0, \\
& \alpha_{3}=-\alpha_{6}=\alpha, \quad \alpha_{4}=\alpha_{5}=\hat{\alpha} .
\end{aligned}
$$

Now, we obtain from (3.8), (3.9) and (3.10) with the correspondent $\ell, r$ superscripts

$$
\begin{aligned}
& \alpha\left[s h-B_{3}\left(D_{2}+B_{3}\right)\right]+\hat{\alpha} B_{3}\left[B_{2}-D_{3}\right]=0, \\
& \alpha\left[s h-D_{2}\left(D_{2}+B_{3}\right)\right]+\hat{\alpha} D_{2}\left[B_{2}-D_{3}\right]=0, \\
& \alpha B_{2}\left[D_{2}+B_{3}\right]-\hat{\alpha}\left[s h-B_{2}\left(B_{2}-D_{3}\right)\right]=0, \\
& \alpha D_{3}\left[D_{2}+B_{3}\right]-\hat{\alpha}\left[s h+D_{3}\left(B_{2}-D_{3}\right)\right]=0 .
\end{aligned}
$$

Thus, a similar argument as above for the columns implies $\alpha=\hat{\alpha}=0$. Analogously, we obtain for $P^{\ell}, P^{r}=1$. Consequently, the matrix $A$ is non-singular.

4. Finally, we show that for any $\tilde{s}$ sufficiently close to $s$, that is, $\left(\tilde{s}, \tilde{u}^{\ell}, \tilde{u}^{r}\right)$ in a neighborhood of $\left(s, u^{\ell}, u^{r}\right)$, we have a solution for the Riemann problem. First, from item 2, we observe that $\left(s, u^{\ell}, u^{r}\right)$ satisfies

$$
\psi\left(s, u^{\ell}, u^{r}\right)=0
$$


Moreover, we recall from item 1 that $\tilde{s}$ must be a rarefaction shock, which is the case for $s$. Now, from item 3 , the matrix $A$ defined as

$$
[A]_{i j}=\partial_{(j+1)} \psi_{i}\left(s, u^{\ell}, u^{r}\right) \quad(i, j=1, \ldots, 8)
$$

is non-singular. Then by the Implicit Function Theorem, there exist a neighborhood $U$ of $\left(s, u^{\ell}, u^{r}\right)$, an open set $S \subset \mathbb{R}$, with $s \in S$, and $\phi \in C^{\infty}\left(S ; \mathbb{R}^{8}\right)$, such that

$$
\left\{\left(s_{0}, u_{0}^{\ell}, u_{0}^{r}\right) \in U: \psi\left(s_{0}, u_{0}^{\ell}, u_{0}^{r}\right)=0\right\}=\left\{\left(s_{0}, \phi\left(s_{0}\right)\right): s_{0} \in S\right\} .
$$

So (1.8)-(1.11) are not complete by themselves; they must be augmented by some selection criteria.

Acknowledgments. Wladimir Neves' research was supported in part by CNPqBrazil, proc. 202351/02-5, FAPERJ, proc. 170439/03, FUJB, proc. 10643-7, and International Cooperation Agreement Brazil-France. The first author would like to thank the staff of UMPA of the ENS de Lyon, and he is grateful for their warm hospitality.

Denis Serre's research is partially funded by the European IHP project HYKE under the contract HPRN-CT-2002-00282.

\section{REFERENCES}

[1] Abeyaratne, R., Knowles, J. K., Kinetic Relations and the Propagation of Phase Boundaries in Solids, Arch. Rational Mech. Anal. 114 (1991), 119-154. MR.1094433 (92a:73006)

[2] Boillat, G., Chocs caractéristiques, C. R. Acad. Sci. Paris, Serie A-B 274 (1972), A1018-A1021. MR0295670(45:4736)

[3] Born, M., Infeld, L., Foundations of a new field theory, Proc. Roy. London, A 144 (1934), 425-451.

[4] Brenier, Y., Hydrodynamic structure of the augmented Born-Infeld equations, Arch. Rational Mech. Anal. 172 (2004), 65-91. MR2048567 (2005a:35264)

[5] Bressan, A., LeFloch, P. Uniqueness of weak solutions to systems of conservation laws, Arch. Rational Mech. Anal. 140 (1997), 301-317. MR1489317 (98m:35125)

[6] Bressan, A., Goatin, P. Oleinik type estimates and uniqueness for $n \times n$ conservation laws, J. Diff. Eq. 156 (1999), 26-49. MR1701818 (2000d:35134)

[7] Bressan, A., Liu, T.-P., Yang, T., $L^{1}$ stability estimates for $n \times n$ conservation laws, Arch. Rational Mech. Anal. 149 (1999), 1-22. MR1723032 (2000g:35139)

[8] Bressan, A., Crasta, G., Piccoli, B., Well-posedness of the Cauchy problem for $n \times n$ systems of conservation laws, Mem. Am. Math. Soc. 146, no. 694 (2000). MR1686652 (2000m:35122)

[9] Coleman, B., D., Dill, E., H. Thermodynamic restrictions on the constitutive equations of electromagnetic theory, Z. Angew. Math. Phys., 22 (1971), 691-702.

[10] Dafermos, C., Hyperbolic Conservation Laws in Continuum Physics, Springer, 2000. MR1763936 (2001m:35212)

[11] Dafermos, C., The second law of thermodynamics and stability, Arch. Rational Mech. Anal. 70 (1979), 167-179. MR0546634 (80j:73004)

[12] DiPerna, R. J., Uniqueness of solutions to hyperbolic conservation laws, Indiana U. Math. J. 28 (1979), 137-188. MR0523630 (80i:35119)

[13] Demoulini, S., Stuart, D., Tzavaras, A., A variational approximation scheme for three-dimensional elastodynamics with polyconvex energy, Arch. Rational Mech. Anal. 157 (2001), 325-344. MR.1831175 (2002b:74025)

[14] Evans, L., C., Gariepy, R., F., Measure Theory and Fine Properties of Functions, CRC Press, Boca Raton, FL, 1992. MR 1158660|(93f:28001)

[15] Fan, H., Slemrod M., Dynamic Flows with Liquid/Vapor Phase Transitions, Handbook of Mathematical Fluid Dynamics, North-Holland, vol. 1 (2002), 373-420. MR1942467 (2003j:76085)

[16] Kulikovskii, A., Sveshnikova, E., Nonlinear Waves in Elastic Media, CRC Press, Boca Raton, FL, 1995. MR:1396086 (97h:73003) 
[17] LeFloch , P. G., Propagation Phase Boundaries: Formulation of the Problem and Existence via the Glimm Method, Arch. Rational Mech. Anal. 123 (1993), 153-197. MF1219421 (94m:35187)

[18] Majda, A., Compressible Fluid Flow and Systems of Conservation Laws in Several Space Variables, New York: Springer, 1984. MR0748308 (85e:35077)

[19] Serre, D., Systems of Conservation Laws, Vols. 1-2, Cambridge: Cambridge University Press, 1999. MR.1707279 (2000g:35142)

[20] Serre, D., Hyperbolicity of the nonlinear models of Maxwell's equations, Archive Ration. Mech. Anal. 172 (2004), 309-331. MR2062427 